\title{
LAMBERT: Layout-Aware Language Modeling for Information Extraction
}

\author{
Łukasz Garncarek ${ }^{1 \star}$, Rafał Powalski ${ }^{1}$, Tomasz Stanisławek ${ }^{1,2}$, \\ Bartosz Topolski ${ }^{1}$, Piotr Halama ${ }^{1}$, Michał Turski ${ }^{1,3}$, and Filip Graliński ${ }^{1,3}$ \\ 1 Applica.ai, Zajęcza 15, 00-351 Warszawa, Poland \\ firstname. lastname@applica.ai \\ 2 Warsaw University of Technology, Koszykowa 75, 00-662 Warszawa, Poland \\ firstname.lastname@pw.edu.pl \\ 3 Adam Mickiewicz University, 1 Wieniawskiego, 61-712 Poznań, Poland \\ firstname. lastname@amu.edu.pl
}

\begin{abstract}
We introduce a simple new approach to the problem of understanding documents where non-trivial layout influences the local semantics. To this end, we modify the Transformer encoder architecture in a way that allows it to use layout features obtained from an OCR system, without the need to re-learn language semantics from scratch. We only augment the input of the model with the coordinates of token bounding boxes, avoiding, in this way, the use of raw images. This leads to a layout-aware language model which can then be fine-tuned on downstream tasks.

The model is evaluated on an end-to-end information extraction task using four publicly available datasets: Kleister NDA, Kleister Charity, SROIE and CORD. We show that our model achieves superior performance on datasets consisting of visually rich documents, while also outperforming the baseline RoBERTa on documents with flat layout (NDA $F_{1}$ increase from 78.50 to 80.42 ). Our solution ranked first on the public leaderboard for the Key Information Extraction from the SROIE dataset, improving the SOTA $F_{1}$-score from 97.81 to 98.17 .
\end{abstract}

Keywords: Language model · Layout - Key information extraction . Transformer · Visually rich document · Document understanding

\section{Introduction}

The sequential structure of text leads to it being treated as a sequence of tokens, characters, or more recently, subword units. In many problems related to Natural Language Processing (NLP), this linear perspective was enough to enable significant breakthroughs, such as the introduction of the neural Transformer architecture [28]. In this setting, the task of computing token embeddings is solved by Transformer encoders, such as BERT [6] and its derivatives, achieving top scores on the GLUE benchmark [29].

* corresponding author; the first four authors have equally contributed to the paper. 
They all deal with problems arising in texts defined as sequences of words. However, in many cases there is a structure more intricate than just a linear ordering of tokens. Take, for instance, printed or richly-formatted documents, where the relative positions of tokens contained in tables, spacing between paragraphs, or different styles of headers, all carry useful information. After all, the goal of endowing texts with layout and formatting is to improve readability.

In this article we present one of the first attempts to enrich the state-of-theart methods of NLP with layout understanding mechanisms, contemporaneous with [32], to which we compare our model. Our approach injects the layout information into a pretrained instance of RoBERTa. We fine-tune the augmented model on a dataset consisting of documents with non-trivial layout.

We evaluate our model on the end-to-end information extraction task, where the training set consists of documents and the target values of the properties to be extracted, without any additional annotations specifying the locations where the information on these properties can be found in the documents. We compare the results with a baseline RoBERTa model, which relies on the sequential order of tokens obtained from the OCR alone (and does not use the layout features), and with the solution of 3231. LAMBERT achieves superior performance on visually rich documents, without sacrificing results on more linear texts.

\subsection{Related work}

There are two main lines of research into understanding documents with nontrivial layout. The first one is Document Layout Analysis (DLA), the goal of which is to identify contiguous blocks of text and other non-textual objects on the page and determine their function and order in the document. The obtained segmentation can be combined with the textual information contained in the detected blocks. This kind of method has recently been employed in [17.

Many services employ DLA functionality for OCR (which requires document segmentation), table detection or form field detection, and their capabilities are still expanding. The most notable examples are Amazon Textract [1, the Google Cloud Document Understanding AI platform [8], and Microsoft Cognitive Services [20]. However, each has limitations, such as the need to create rules for extracting information from the tables recognized by the system, or use training datasets with annotated document segments. More recent works on information extraction using DLA include, among others, [14|3|10|2|19|22 25. They concentrate on specific types of documents, such as invoices or forms, where the layout plays a relatively greater role: more general documents may contain tables, but they can also have large amounts of unstructured text.

The second idea is to directly combine the methods of Computer Vision and NLP. This could be done, for instance, by representing a text-filled page as a multi-channel image, with channels corresponding to the features encoding the semantics of the underlying text, and, subsequently, using convolutional networks. This method was used, among others, by Chargrid and BERTgrid models [15|5]. On the other hand, LayoutLM [32] and TRIE [34] used the image recognition features of the page image itself. A more complex approach was taken 
by PICK [33], which separately processes the text and images of blocks identified in the document. In this way it computes the vertex embeddings of the block graph, which is then processed with a graph neural network.

Our idea is also related to the one used in [24], though in a different setting. They considered texts accompanied by audio-visual signal injected into a pretrained BERT instance, by combining it with the input embeddings.

LAMBERT has a different approach. It uses neither the raw document image, nor the block structure that has to be somehow inferred. It relies on the tokens and their bounding boxes alone, both of which are easily obtainable from any reasonable OCR system.

\subsection{Contribution}

Our main contribution is the introduction of a Layout-Aware Language Model, a general-purpose language model that views text not simply as a sequence of words, but as a collection of tokens on a two-dimensional page. As such it is able to process plain text documents, but also tables, headers, forms and various other visual elements. The implementation of the model is available at https://github.com/applicaai/lambert.

A key feature of this solution is that it retains the crucial trait of language models: the ability to learn in an unsupervised setting. This allows the exploitation of abundantly available unannotated public documents, and a transfer of the learned representations to downstream tasks. Another advantage is the simplicity of this approach, which requires only an augmentation of the input with token bounding boxes. In particular, no images are needed. This eliminates an important performance factor in industrial systems, where large volumes of documents have to be sent over a network between distributed processing services.

Another contribution of the paper is an extensive ablation study of the impact of augmenting RoBERTa with various types of additional positional embeddings on model performance on the SROIE [12, CORD [21], Kleister NDA and Kleister Charity datasets [27.

Finally, we created a new dataset for the unsupervised training of layoutaware language models. We will share a 200k document subset, amounting to $2 \mathrm{M}$ visually rich pages, accompanied by a dual classification of documents: business/legal documents with complex structure; and others. Due to IIT-CDIP Test Collection dataset [16] accessibility problems this would constitute the largest widely available dataset for training layout-aware language models. It would allow researchers to compare the performance of their solutions not only on the same test sets, but also with the same training set. The dataset is published at https://github.com/applicaai/lambert, together with a more detailed description that is too long for this paper.

\footnotetext{
${ }^{4}$ the link https://ir.nist.gov/cdip/ seems to be dead (access on Feb 17, 2021)
} 


\section{Proposed method}

We inject the layout information into the model in two ways. Firstly, we modify the input embeddings of the original RoBERTa model by adding the layout term. We also experiment with completely removing the sequential embedding term. Secondly, we apply relative attention bias, used in 2611/23 in the context of sequential position. The final architecture is depicted in Figure 1

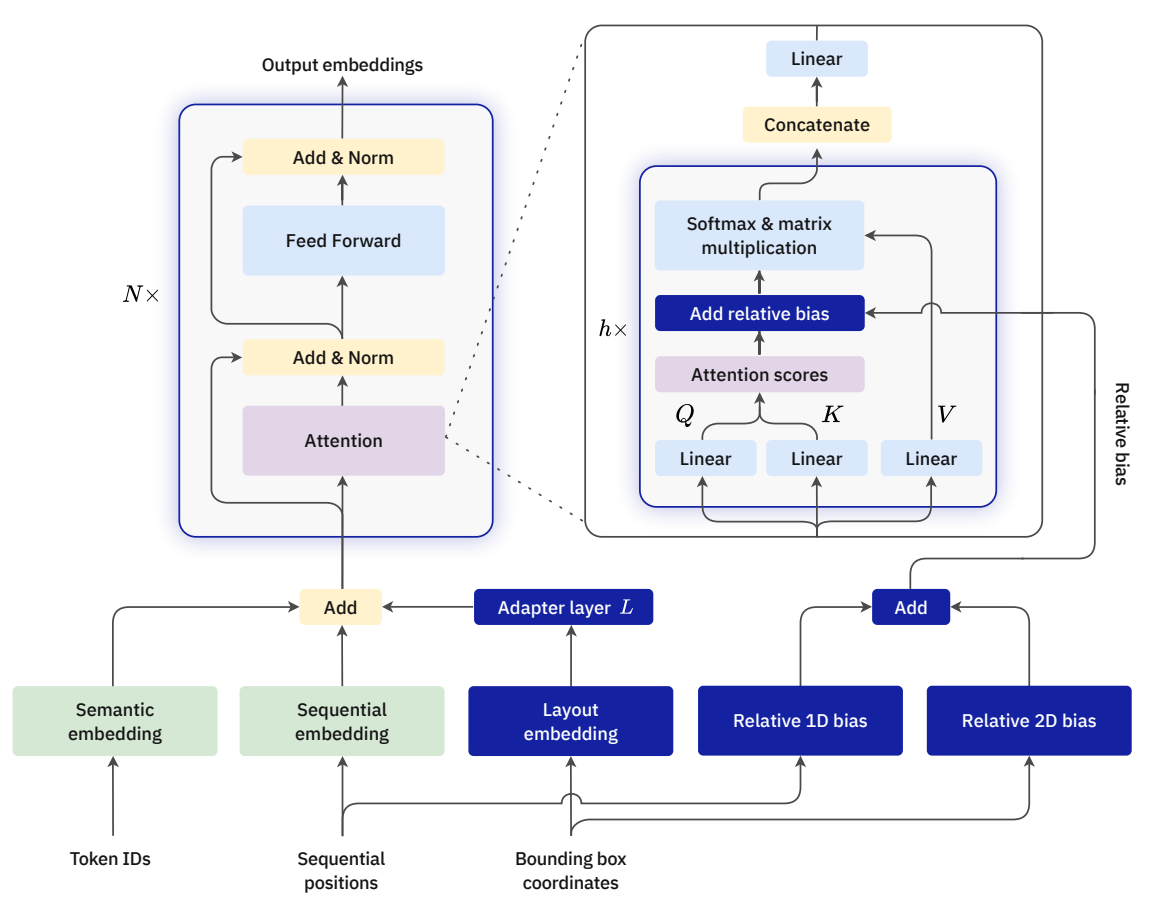

Fig. 1: LAMBERT model architecture. Differences with the plain RoBERTa model are indicated by white text on dark blue background. $N=12$ is the number of transformer encoder layers, and $h=12$ is the number of attention heads in each encoder layer. $Q, K$, and $V$ are, respectively, the queries, keys and values obtained by projecting the self-attention inputs.

\subsection{Background}

The basic Transformer encoder, used in, for instance, BERT [6] and RoBERTa [18, is a sequence-to-sequence model transforming a sequence of input embeddings $x_{i} \in \mathbb{R}^{n}$ into a sequence of output embeddings $y_{i} \in \mathbb{R}^{m}$ of the same length, 
for the input/output dimensions $n$ and $m$. One of the main distinctive features of this architecture is that it discards the order of its input vectors. This allows parallelization levels unattainable for recurrent neural networks.

In such a setting, the information about the order of tokens is preserved not by the structure of the input. Instead, it is explicitly passed to the model, by defining the input embeddings as

$$
x_{i}=s_{i}+p_{i},
$$

where $s_{i} \in \mathbb{R}^{n}$ is the semantic embedding of the token at position $i$, taken from a trainable embedding layer, while $p_{i} \in \mathbb{R}^{n}$ is a positional embedding, depending only on $i$. In order to avoid confusion, we will, henceforth, use the term sequential embeddings instead of positional embeddings, as the positional might be understood as relating to the 2-dimensional position on the page, which we will deal with separately.

Since in RoBERTa, on which we base our approach, the embeddings $p_{i}$ are trainable, the number of pretrained embeddings (in this case 512) defines a limit on the length of the input sequence. In general, there are many ways to circumvent this limit, such as using predefined 28] or relative [4 sequential embeddings.

\subsection{Modification of input embeddings}

We replace the input embeddings defined in (1) with

$$
x_{i}=s_{i}+p_{i}+L\left(\ell_{i}\right) .
$$

Here, $\ell_{i} \in \mathbb{R}^{k}$ stands for layout embeddings, which are described in detail in the next subsection. They carry the information about the position of the $i$-th token on the page.

The dimension $k$ of the layout embeddings is allowed to differ from the input embedding dimension $n$, and this difference is dealt with by a trainable linear layer $L: \mathbb{R}^{k} \rightarrow \mathbb{R}^{n}$. However, our main motivation to introduce the adapter layer $L$ was to gently increase the strength of the signal of layout embeddings during training. In this way, we initially avoided presenting the model with inputs that it was not prepared to deal with. Moreover, in theory, in the case of non-trainable layout embeddings, the adapter layer may be able to learn to project $\ell_{i}$ onto a subspace of the embedding space that reduces interference with the other terms in (2). For instance, it is possible for the image of the adapter layer to learn to be approximately orthogonal to the sum of the remaining terms. This would minimize any information loss caused by adding multiple vectors. While this was our theoretical motivation, and it would be interesting to investigate in detail how much of it actually holds, such detailed considerations of a single model component exceed the scope of this paper. We included the impact of using the adapter layer in the ablation study.

We initialize the weight matrix of $L$ according to a normal distribution $\mathcal{N}\left(0, \sigma^{2}\right)$, with the standard deviation $\sigma$ being a hyperparameter. We have to 
choose $\sigma$ carefully, so that in the initial phase of training, the $L\left(\ell_{i}\right)$ term does not interfere overly with the already learned representations. We experimentally determined the value $\sigma=0.02$ to be near-optima $\left.\right|^{5}$

\subsection{Layout embeddings}

In our setting, a document is represented by a sequence of tokens $t_{i}$ and their bounding boxes $b_{i}$. To each element of this sequence, we assign its layout embedding $\ell_{i}$, carrying the information about the position of the token with respect to the whole document. This could be performed in various ways. What they all have in common is that the embeddings $\ell_{i}$ depend only on the bounding boxes $b_{i}$ and not on the tokens $t_{i}$.

We base our layout embeddings on the method originally used in [7], and then in $[28$ to define the sequential embeddings. We first normalize the bounding boxes by translating them so that the upper left corner is at $(0,0)$, and dividing their dimensions by the page height. This causes the page bounding box to become $(0,0, w, 1)$, where $w$ is the normalized width.

The layout embedding of a token will be defined as the concatenation of four embeddings of the individual coordinates of its bounding box. For an integer $d$ and a vector of scaling factors $\theta \in \mathbb{R}^{d}$, we define the corresponding embedding of a single coordinate $t$ as

$$
\operatorname{emb}_{\theta}(t)=(\sin (t \theta) ; \cos (t \theta)) \in \mathbb{R}^{2 d},
$$

where the sin and cos are performed element-wise, yielding two vectors in $\mathbb{R}^{d}$. The resulting concatenation of single bounding box coordinate embeddings is then a vector in $\mathbb{R}^{8 d}$.

In [28, Section 3.5], and subsequently in other Transformer-based models with precomputed sequential embeddings, the sequential embeddings were defined by $\operatorname{emb}_{\theta}$ with $\theta$ being a geometric progression interpolating between 1 and $10^{-4}$. Unlike the sequential position, which is a potentially large integer, bounding box coordinates are normalized to the interval $[0,1]$. Hence, for our layout embeddings we use larger scaling factors $\left(\theta_{r}\right)$, namely a geometric sequence of length $n / 8$ interpolating between 1 and 500 , where $n$ is the dimension of the input embeddings.

\subsection{Relative bias}

Let us recall that in a typical Transformer encoder, a single attention head transforms its input vectors into three sequences: queries $q_{i} \in \mathbb{R}^{d}$, keys $k_{i} \in \mathbb{R}^{d}$, and values $v_{i} \in \mathbb{R}^{d}$. The raw attention scores are then computed as $\alpha_{i j}=$ $d^{-1 / 2} q_{i}^{T} k_{j}$. Afterwards, they are normalized using softmax, and used as weights in linear combinations of value vectors.

The point of relative bias is to modify the computation of the raw attention scores by introducing a bias term: $\alpha_{i j}^{\prime}=\alpha_{i j}+\beta_{i j}$. In the sequential setting,

\footnotetext{
${ }^{5}$ we tested the values $0.5,0.1,0.02,0.004$, and 0.0008
} 
$\beta_{i j}=W(i-j)$ is a trainable weight, depending on the relative sequential position of tokens $i$ and $j$. This form of attention bias was introduced in [23], and we will refer to it as sequential attention bias.

We introduce a simple and natural extension of this mechanism to the twodimensional context. In our case, the bias $\beta_{i j}$ depends on the relative positions of the tokens. More precisely, let $C \gg 1$ be an integer resolution factor (the number of cells in a grid used to discretize the normalized coordinates). If $b_{i}=$ $\left(x_{1}, y_{1}, x_{2}, y_{2}\right)$ is the normalized bounding box of the $i$-th token, we first reduce it to a 2 -dimensional position $\left(\xi_{i}, \eta_{i}\right)=\left(C x_{1}, C\left(y_{1}+y_{2}\right) / 2\right)$, and then define

$$
\beta_{i j}=H\left(\left\lfloor\xi_{i}-\xi_{j}\right\rfloor\right)+V\left(\left\lfloor\eta_{i}-\eta_{j}\right\rfloor\right),
$$

where $H(\ell)$ and $V(\ell)$ are trainable weights defined for every integer $\ell \in[-C, C)$. A good value for $C$ should allow for a distinction between consecutive lines and tokens, without unnecessarily affecting performance. For a typical document $C=100$ is enough, and we fix this in our experiments.

This form of attention bias will be referred to as $2 D$ attention bias. We suspect that it should help in analyzing, say, tables by allowing the learning of relationships between cells.

\section{Experiments}

All experiments were performed on 8 NVIDIA Tesla V100 32GB GPUs. As our pretrained base model we used RoBERTa in its smaller, base variant (125M parameters, 12 layers, 12 attention heads, hidden dimension 768). This was also employed as the baseline, after additional training on the same dataset we used for LAMBERT. The implementation and pretrained weights from the transformers library [30] were used.

In the LAMBERT model, we used the layout embeddings of dimension $k=$ 128 , and initialized the adapter layer $L$ with standard deviation $\sigma=0.02$, as noted in Section 2 For comparison, in our experiments, we also included the published version of the LayoutLM model [32, which is of a similar size.

The models were trained on a masked language modeling objective extended with layout information (with the same settings as the original RoBERTa [18]); and subsequently, on downstream information extraction tasks. In the remainder of the paper, these two stages will be referred to as, respectively, training and fine-tuning.

Training was performed on a collection of PDFs extracted from Common Crawl made up of a variety of documents (we randomly selected up to 10 documents from any single domain). The documents were processed with an OCR system, Tesseract 4.1.1-rc1-7-gb36c, to obtain token bounding boxes. The final model was trained on the subset of the corpus consisting of business documents with non-trivial layout, filtered by an SVM binary classifier, totaling to approximately $315 \mathrm{k}$ documents (3.12M pages). The SVM model was trained on 700 manually annotated PDF files to distinguish between business (e.g. invoices, forms) and non-business documents (e.g. poems, scientific texts). 
In the training phase, we used the Adam optimizer with the weight decay fix from [30]. We employed a learning rate scheduling method similar to the one used in 6, increasing the learning rate linearly from 0 to $1 \mathrm{e}-4$ for the warm-up period of $10 \%$ of the training time and then decreasing it linearly to 0 . The final model was trained with batch size of 128 sequences (amounting to $64 \mathrm{~K}$ tokens) for approximately 1000k steps (corresponding to training on $3 \mathrm{M}$ pages for 25 epochs). This took about 5 days to complete a single experiment.

After training our models, we fine-tuned and evaluated them independently on multiple downstream end-to-end information extraction tasks. Each evaluation dataset was split into training, validation and test subsets. The models were extended with a simple classification head on top, consisting of a single linear layer, and fine-tuned on the task of classifying entity types of tokens. We employed early stopping based on the $F_{1}$-score achieved on the validation part of the dataset. We used the Adam optimizer again, but this time without the learning rate warm-up, as it turned out to have no impact on the results.

The extended model operates as a tagger on the token level, allowing for the classification of separate tokens, while the datasets contain only the values of properties that we are supposed to extract from the documents. Therefore, the further processing of output is required. To this end, we use the pipeline described in [27.

Every contiguous sequence of tokens tagged as a given entity type is treated as a recognized entity and assigned a score equal to the geometric mean of the scores of its constituent tokens. Then, every recognized entity undergoes a normalization procedure specific to its general data type (e.g. date, monetary amount, address, etc.). This is performed using regular expressions: for instance, the date July, 15th 2013 is converted to 2013-07-15. Afterwards, duplicates are aggregated by summing their scores, leading to a preference for entities detected multiple times. Finally, the highest-scoring normalized entity is selected as the output of the information extraction system. The predictions obtained this way are compared with target values provided in the dataset using $F_{1}$-score as the evaluation metric. See [27] for more details.

\section{Results}

We evaluated our models on four public datasets containing visually rich documents. The Kleister NDA and Kleister Charity datasets are part of a larger Kleister dataset, recently made public in 27] (many examples of documents, and detailed descriptions of extraction tasks can be found therein). The NDA set consists of legal agreements, whose layout variety is limited. It should probably be treated as a plain-text dataset. The Charity dataset on the other hand contains reports of UK charity organizations, which include various tables, diagrams and other graphic elements, interspersed with text passages. All Kleister datasets come with predefined train/dev/test splits, with 254/83/203 documents for NDA and 1729/440/609 for Charity. 
Table 1: Comparison of $F_{1}$-scores for the considered models. Best results in each column are indicated in bold. In parentheses, the length of training of our models, expressed in non-unique pages, is presented for comparison. For RoBERTa, the first row corresponds to the original pretrained model without any further training, while in the second row the model was trained on our dataset. ${ }^{a}$ result obtained from relevant publication; ${ }^{b}$ result of a single model, obtained from the SROIE leaderboard [13]

\begin{tabular}{|c|c|c|c|c|c|c|c|}
\hline \multirow{2}{*}{ Model } & \multirow{2}{*}{ Params } & \multicolumn{4}{|c|}{ Our experiments } & \multicolumn{2}{|c|}{ External results } \\
\hline & & $\mathrm{NDA}$ & Charity & SROIE* & CORD & SROIE & CORD \\
\hline RoBERTa 18 & $125 \mathrm{M}$ & 77.91 & 76.36 & 94.05 & 91.57 & $92.39^{\mathrm{b}}$ & - \\
\hline RoBERTa (16M) & $125 \mathrm{M}$ & 78.50 & 77.88 & 94.28 & 91.98 & $93.03^{\mathrm{b}}$ & - \\
\hline \multirow{2}{*}{ LayoutLM 32 . } & $113 \mathrm{M}$ & 77.50 & 77.20 & 94.00 & 93.82 & $94.38^{\mathrm{a}}$ & $94.72^{\mathrm{a}}$ \\
\hline & $343 \mathrm{M}$ & 79.14 & 77.13 & 96.48 & 93.62 & $97.09^{\mathrm{b}}$ & $94.93^{\mathrm{a}}$ \\
\hline \multirow{2}{*}{ LayoutLMv2 31 } & $200 \mathrm{M}$ & - & - & - & - & $96.25^{\mathrm{a}}$ & $94.95^{\mathrm{a}}$ \\
\hline & $426 \mathrm{M}$ & - & - & - & - & $97.81^{\mathrm{b}}$ & $96.01^{\mathrm{a}}$ \\
\hline LAMBERT (16M) & $125 \mathrm{M}$ & 80.31 & 79.94 & 96.24 & 93.75 & - & - \\
\hline LAMBERT (75M) & $125 \mathrm{M}$ & 80.42 & 81.34 & 96.93 & 94.41 & $98.17^{\mathrm{b}}$ & - \\
\hline
\end{tabular}

The SROIE [12] and CORD [21] datasets are composed of scanned and OCRed receipts. Documents in SROIE are annotated with four target entities to be extracted, while in CORD there are 30 different entities. We use the public 1000 samples from the CORD dataset with the train/dev/test split proposed by the authors of the dataset (respectively, 800/100/100). As for SROIE, it consists of a public training part, and test part with unknown annotations. For the purpose of ablation studies, we further subdivided the public part of SROIE into training and test subsets (546/80 documents; due to the lack of a validation set in this split, we fine-tuned for 15 epochs instead of employing early stopping). We refer to this split as SROIE*, while the name SROIE is reserved for the original SROIE dataset, where the final evaluation on the test set is performed through the leaderboard [13].

In Table 1, we present the evaluation results achieved on downstream tasks by the trained models. With the exception of the Kleister Charity dataset, where only 5 runs were made, each of the remaining experiments were repeated 20 times, and the mean result was reported. We compare LAMBERT with baseline RoBERTa (trained on our dataset) and the original RoBERTa 18] (without additional training); LayoutLM [32]; and LayoutLMv2 31. The LayoutLM model published by its authors was plugged into the same pipeline that we used for LAMBERT and RoBERTa. In the first four columns we present averaged results of our experiments, and for CORD and SROIE we additionally provide the results reported by the authors of LayoutLM, and presented on the leaderboard [13]. 
Since the LayoutLMv2 model was not publicly available at the time of preparing this article, we could not perform experiments ourselves. As a result some of the results are missing. For CORD, we present the scores given in [31], where the authors did not mention, though, whether they averaged over multiple runs, or used just a single model. A similar situation occurs for LayoutLM; we presented the average results of 20 runs (best run of LAMBERT attained the score of 95.12), which are lower than the scores presented in 31. The difference could be attributed to using a different end-to-end evaluation pipeline, or averaging (if the results in [31 32] come from a single run).

For the full SROIE dataset, most of the results were retrieved from the public leaderboard [13], and therefore they come from a single model. For the base variants of LayoutLM and LayoutLMv2, the results were unavailable, and we present the scores from the corresponding papers.

In our experiments, the base variant of LAMBERT achieved top scores for all datasets. However, in the case of CORD, the result reported in [31] for the large variant of LayoutLMv2 is superior. If we consider the best scores of LAMBERT (95.12) instead of the average, and the scores of LayoutLM reported in 32, LAMBERT slightly outperforms LayoutLM, while still being inferior to LayoutLMv2. Due to the lack of details on the results of LayoutLM, it is unknown which of these comparisons is valid.

For Kleister datasets, the base variant (and in the case of Charity, also the large variant) of LayoutLM did not outperform the baseline RoBERTa. We suspect that this might be the result of LayoutLM being better attuned to the evaluation pipeline used by its authors, and the fact that it was based on an uncased language model. In the Kleister dataset, meanwhile, performance for entities such as names may depend on casing.

\section{$5 \quad$ Hyperparameters and ablation studies}

In order to investigate the impact of our modifications to RoBERTa, we performed an extensive study of hyperparameters and the various components of the final model. We investigated the dimension of layout embeddings, the impact of the adapter layer $L$, the size of training dataset, and finally we performed a detailed ablation study of the embeddings and attention biases we had used to augment the baseline model.

In the studies, every model was fine-tuned and evaluated 20 times on each dataset, except for Kleister Charity dataset, on which we fine-tuned every model 5 times: evaluations took much longer on Kleister Charity. For each model and dataset combination, the mean score was reported, together with the two-sided 95\% confidence interval, computed using the corresponding $t$-value. We considered differences to be significant when the corresponding intervals were disjoint. All the results are presented in Table 2, which is divided into sections corresponding to different studies. The $F_{1}$-scores are reported as increases with respect to the reported mean baseline score, to improve readability. 
Table 2: Improvements of $F_{1}$-score over the baseline for various variants of LAMBERT model. The first row (with grayed background) contains the $F_{1}$-scores of the baseline RoBERTa model. The other grayed row corresponds to full LAMBERT. Statistically insignificant improvements over the baseline are grayed. In each of three studies, the best result together with all results insignificantly smaller are in bold. ${ }^{a}$ filtered datasets; ${ }^{\mathrm{b}}$ model with a disabled adapter layer

\begin{tabular}{|c|c|c|c|c|c|c|c|c|c|}
\hline \multirow{2}{*}{ 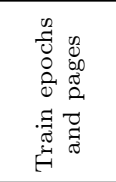 } & \multirow[b]{2}{*}{ 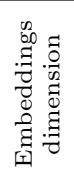 } & \multicolumn{4}{|c|}{ Inputs } & \multicolumn{4}{|c|}{ Datasets } \\
\hline & & 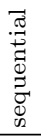 & 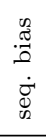 & $\begin{array}{l}\vec{z} \\
\stackrel{0}{0} \\
\stackrel{\Xi}{\sigma}\end{array}$ & $\begin{array}{l}\frac{0}{0} \\
\stackrel{0}{0} \\
\text { a }\end{array}$ & NDA & Charity & SROIE* & CORD \\
\hline \multirow{3}{*}{$8 \times 2 \mathrm{M}$} & \multirow{3}{*}{128} & • & $\bullet$ & & & $\begin{array}{r}78.50_{ \pm 1} .16 \\
\mathbf{1 . 9 4}_{ \pm 0.46} \\
\mathbf{2 . 4 2} \pm 0.61 \\
\end{array}$ & $\begin{array}{r}77.88 \pm 0.48 \\
-0.82 \pm 0.74 \\
0.52 \pm 0.64 \\
\end{array}$ & $\begin{array}{r}94.28 \pm 0.42 \\
0.33 \pm 0.22 \\
0.79_{ \pm 0.17} \\
\end{array}$ & $\begin{array}{r}91.98 \pm 0.62 \\
-0.15 \pm 0.49 \\
0.03 \pm 0.57 \\
\end{array}$ \\
\hline & & & & $\bullet$ & $\bullet$ & $\begin{array}{r}1.25 \pm 0.59 \\
-0.49 \pm 0.62 \\
0.88 \pm 0.50\end{array}$ & $\begin{array}{r}\mathbf{2 . 6 2}_{ \pm 0.80} \\
2.02_{ \pm 0.48} \\
\mathbf{3 . 0 0}_{ \pm 0.37}\end{array}$ & $\begin{array}{r}\mathbf{1 . 8 6} \pm 0.15 \\
0.53 \pm 0.28 \\
\mathbf{1 . 9 4}_{ \pm 0.16}\end{array}$ & $\begin{array}{r}0.89 \pm 0.83 \\
-0.17 \pm 0.62 \\
0.68 \pm 0.62\end{array}$ \\
\hline & & $\begin{array}{l}\dot{ } \\
\bullet \\
\bullet \\
\bullet \\
\bullet\end{array}$ & $\begin{array}{l}\bullet \\
\bullet\end{array}$ & $\begin{array}{l}\bullet \\
\bullet \\
\bullet \\
\bullet\end{array}$ & $\begin{array}{l}\bullet \\
\bullet \\
\bullet \\
\bullet\end{array}$ & $\begin{array}{l}1.74 \pm 0.67 \\
1.73 \pm 0.60 \\
0.54 \pm 0.85 \\
1.66 \pm 0.76 \\
0.85 \pm 0.91 \\
\mathbf{1 . 8 1} \pm 0.60\end{array}$ & $\begin{array}{r}0.06 \pm 0.93 \\
2.02_{ \pm 0.53} \\
1.84_{ \pm 0.42} \\
0.32_{ \pm 1.35} \\
1.84_{ \pm 0.27} \\
\mathbf{2 . 0 6} 6_{ \pm 0.69}\end{array}$ & $\begin{array}{l}\mathbf{1 . 9 4}_{ \pm 0.18} \\
\mathbf{2 . 0 9}_{ \pm 0.22} \\
\mathbf{2 . 0 8}_{ \pm 0.38} \\
\mathbf{1 . 7 5}_{ \pm 0.35} \\
\mathbf{2 . 0 1}_{ \pm 0.24} \\
\mathbf{1 . 9 6}_{ \pm 0.16}\end{array}$ & $\begin{array}{r}\mathbf{1 . 4 2}_{ \pm 0.53} \\
\mathbf{1 . 9 3}_{ \pm 0.71} \\
\mathbf{2 . 1 5} \pm 0.65 \\
1.06_{ \pm 0.54} \\
\mathbf{1 . 9 5}_{ \pm 0.46} \\
\mathbf{1 . 7}_{ \pm 0.46}\end{array}$ \\
\hline $8 \times 2 \mathrm{M}$ & $\begin{array}{c}128 \\
384 \\
768 \\
768^{\mathrm{b}}\end{array}$ & $\begin{array}{l}\bullet \\
\bullet \\
\bullet \\
\bullet\end{array}$ & & $\begin{array}{l}\bullet \\
\bullet \\
\bullet \\
\bullet\end{array}$ & & $\begin{array}{l}1.74 \pm 0.67 \\
0.90 \pm 0.54 \\
0.71 \pm 1.04 \\
0.77 \pm 0.58\end{array}$ & $\begin{array}{r}0.06 \pm 0.93 \\
0.70 \pm 0.40 \\
0.50_{ \pm 0.85} \\
\mathbf{2 . 3 0} \pm 0.20\end{array}$ & 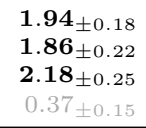 & $\begin{array}{l}\mathbf{1 . 4 2}_{ \pm 0.53} \\
\mathbf{1 . 5 1}_{ \pm 0.60} \\
\mathbf{1 . 5 4}_{ \pm 0.51} \\
\mathbf{1 . 5 8}_{ \pm 0.52}\end{array}$ \\
\hline $\begin{array}{c}8 \times 2 \mathrm{M} \\
8 \times 2 \mathrm{M}^{\mathrm{a}} \\
25 \times 3 \mathrm{M}^{\mathrm{a}}\end{array}$ & 128 & : & • & $\begin{array}{l}\bullet \\
\bullet\end{array}$ & • & 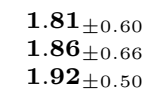 & $\begin{array}{r}2.06_{ \pm 0.26} \\
1.92_{ \pm 0.19} \\
\mathbf{3 . 4 6}_{ \pm 0.21}\end{array}$ & 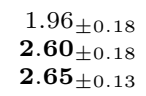 & $\begin{array}{l}1.77_{ \pm 0.46} \\
1.59_{ \pm 0.61} \\
\mathbf{2 . 4 3}_{ \pm 0.19}\end{array}$ \\
\hline
\end{tabular}

\subsection{Baseline}

As a baseline for the studies we use the publicly available pretrained base variant of the RoBERTa model with 12 layers, 12 attention heads, and hidden dimension 768. We additionally trained this model on our training set, and fine-tuned it on the evaluation datasets in a manner analogous to LAMBERT.

\subsection{Embeddings and biases}

In this study we disabled various combinations of input embeddings and attention biases. The models were trained on $2 \mathrm{M}$ pages for 8 epochs, with 128dimensional layout embeddings (if enabled). The resulting models were divided into three groups. The first one contains sequential-only combinations which do not employ the layout information at all, including the baseline. The second group consists of models using only the bounding box coordinates, with no access to sequential token positions. Finally, the models in the third group use both sequential and layout inputs. In this group we did not disable the sequen- 
tial embeddings. It includes the full LAMBERT model, with all embeddings and attention biases enabled.

Generally, we observe that none of the modifications has led to a significant performance deterioration. Among the models considered, the only one which reported a significant improvement for all four datasets - and at the same time, the best improvement - was the full LAMBERT.

For the Kleister datasets the variance in results was relatively higher than in the case of SROIE* and CORD. This led to wider confidence intervals, and reduced the number of significant outcomes. This is true especially for the Kleister NDA dataset, which is the smallest one. In Kleister NDA, significant improvements were achieved for both sequential-only models, and for full LAMBERT. The differences between these increases were insignificant. It would seem that, for sequential-only models, the sequential attention bias is responsible for the improvement. But after adding the layout inputs, it no longer leads to improvements when unaccompanied by other modifications. Still, achieving better results on sequential-only inputs may be related to the plain text nature of the Kleister NDA dataset.

While other models did not report significant improvement over the baseline, there are still some differences between them to be observed. The model using only $2 \mathrm{D}$ attention bias is significantly inferior to most of the others. This seems to agree with the intuition that relative 2D positions are the least suitable way to pass positional information about plain text.

In the case of the Kleister Charity dataset, significant improvements were achieved by all layout-only models, and all models using the $2 \mathrm{D}$ attention bias. Best improvement was attained by full LAMBERT, and two layout-only models using the layout embeddings; the 2D attention bias used alone improved the results significantly, but did not reach the top score. The confidence intervals are too wide to offer further conclusions, and many more experiments will be needed to increase the significance of the results.

For the SROIE* dataset, except for two models augmented only with a single attention bias, all improvements proved significant. Moreover, the differences between all the models using layout inputs are insignificant. We may conclude that passing bounding box coordinates in any way, except through 2D attention bias, significantly improves the results. As to the lack of significant improvements for $2 \mathrm{D}$ attention bias, we hypothesize that this is due to its relative nature. In all other models the absolute position of tokens is somehow known, either through the layout embeddings, or the sequential position. When a human reads a receipt, the absolute position is one of the main features used to locate the typical positions of entities.

For CORD, which is the more complex of the two receipt datasets, significant improvements were observed only for combined sequential and layout models. In this group, the model using both sequential and layout embeddings, augmented with sequential attention bias, did not yield a significant improvement. There were no significant differences among the remaining models in the group. Con- 
trary to the case of SROIE*, none of the layout-only models achieved significant improvement.

\subsection{Layout embedding dimension}

In this study we evaluated four models, using both sequential and layout embeddings, varying the dimension of the latter. We considered 128-, 384-, and 768-dimensional embeddings. Since this is the same as for the input embeddings of RoBERTaBASE, it was possible to remove the adapter layer $L$, and treat this as another variant, in Table 2 denoted as $768^{\mathrm{b}}$.

In Kleister NDA, there were no significant differences between any of the evaluated models, and no improvements over the baseline. On the other hand, in Kleister Charity, disabling the adapter layer and using the 768-dimensional layout embeddings led to significantly better performance. These results remain consistent with earlier observations that in Kleister NDA the best results were achieved by sequential-only models, while in the case of Kleister Charity, by layout-only models. It seems that in the case of NDA the performance is influenced mostly by the sequential features, while in the case of Charity, removing the adapter layer increases the strength of the signal of the layout embeddings, carrying the layout features which are the main factor affecting performance.

In SROIE* and CORD all results were comparable, with one exception, namely in SROIE*, the model with the disabled adapter layer did not, unlike the remaining models, perform significantly better than the baseline.

\subsection{Training dataset size}

In this study, following the observations from [9], we considered models trained on 3 different datasets. The first model was trained for 8 epochs on $2 \mathrm{M}$ unfiltered (see Section 3 for more details of the filtering procedure) pages. In the second model, we used the same training time and dataset size, but this time only filtered pages were used. Finally, the third model was trained for 25 epochs on $3 \mathrm{M}$ filtered pages.

It is not surprising that increasing the training time and dataset size, leads to an improvement in results, at least up to a certain point. In the case of Kleister NDA dataset, there were no significant differences in the results. For Kleister Charity, the best result was achieved for the largest training dataset, with $75 \mathrm{M}$ filtered pages. This result was also significantly better than the outcomes for the smaller dataset. In the case of SROIE* the two models trained on datasets with filtered documents achieved a significantly higher score than the one trained on unfiltered documents. There was, in fact, no significant difference between these two models. This supports the hypothesis that, in this case, filtering could be the more important factor. Finally, for CORD the situation is similar to Kleister Charity. 


\section{Conclusions and further research}

We introduced LAMBERT, a layout-aware language model, producing contextualized token embeddings for tokens contained in formatted documents. The model can be trained in an unsupervised manner. For the end user, the only difference with classical language models is the use of bounding box coordinates as additional input. No images are needed, which makes this solution particularly simple, and easy to include in pipelines that are already based on OCR-ed documents.

The LAMBERT model outperforms the baseline RoBERTa on information extraction from visually rich documents, without sacrificing performance on documents with a flat layout. This can be clearly seen in the results for the Kleister NDA dataset. Its base variant with around $125 \mathrm{M}$ parameters is also able to compete with the large variants of LayoutLM (343M parameters) and LayoutLMv2 (426M parameters), with Kleister and SROIE datasets achieving superior re-

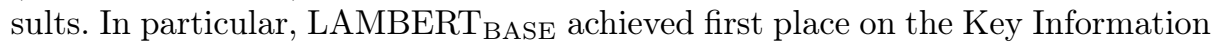
Extraction from the SROIE dataset leaderboard [13].

The choice of particular LAMBERT components is supported by an ablation study including confidence intervals, and is shown to be statistically significant. Another conclusion from this study is that for visually rich documents the point where no more improvement is attained by increasing the training set has not yet been reached. Thus, LAMBERT's performance can still be improved upon by simply increasing the unsupervised training set. In the future we plan to experiment with increasing the model size, and training datasets.

Further research is needed to ascertain the impact of the adapter layer $L$ on the model performance, as the results of the ablation study were inconclusive. It would also be interesting to understand whether the mechanism through which it affects the results is consistent with the hypotheses formulated in Section 2.

Acknowledgments. The authors would like to acknowledge the support the Applica.ai project has received as being co-financed by the European Regional Development Fund (POIR.01.01.01-00-0605/19-00).

\section{References}

1. Amazon: Amazon Textract. https://aws.amazon.com/textract/ (accessed November 25, 2019) (2019)

2. Bart, E., Sarkar, P.: Information extraction by finding repeated structure. In: DAS '10 (2010)

3. Cesarini, F., Francesconi, E., Gori, M., Soda, G.: Analysis and understanding of multi-class invoices. IJDAR 6, 102-114 (2003)

4. Dai, Z., Yang, Z., Yang, Y., Carbonell, J., Le, Q.V., Salakhutdinov, R.: Transformer-XL: Attentive language models beyond a fixed-length context. In: ACL (2019) 
5. Denk, T.I., Reisswig, C.: BERTgrid: Contextualized Embedding for 2D Document Representation and Understanding. In: Workshop on Document Intelligence at NeurIPS 2019 (2019)

6. Devlin, J., Chang, M.W., Lee, K., Toutanova, K.: BERT: Pre-training of deep bidirectional transformers for language understanding. In: NAACL-HLT (2019)

7. Gehring, J., Auli, M., Grangier, D., Yarats, D., Dauphin, Y.N.: Convolutional sequence to sequence learning. In: ICML (2017)

8. Google: Cloud Document Understanding AI. https://cloud.google.com/docum ent-understanding/docs/ (accessed November 25, 2019) (2019)

9. Gururangan, S., Marasović, A., Swayamdipta, S., Lo, K., Beltagy, I., Downey, D., Smith, N.A.: Don't stop pretraining: Adapt language models to domains and tasks. In: Proceedings of the 58th Annual Meeting of the Association for Computational Linguistics. pp. 8342-8360. Association for Computational Linguistics (2020). https://doi.org/10.18653/v1/2020.acl-main.740

10. Hamza, H., Belaïd, Y., Belaïd, A., Chaudhuri, B.: An end-to-end administrative document analysis system. In: 2008 The Eighth IAPR International Workshop on Document Analysis Systems. pp. 175-182 (2008)

11. Huang, Y., Cheng, Y., Bapna, A., Firat, O., Chen, M., Chen, D., Lee, H., Ngiam, J., Le, Q.V., Wu, Y., Chen, Z.: Gpipe: Efficient training of giant neural networks using pipeline parallelism. In: NeurIPS (2019)

12. ICDAR: Competition on Scanned Receipts OCR and Information Extraction. ht tps://rrc.cvc.uab.es/?ch=13 (accessed February 21, 2021) (2019)

13. ICDAR: Leaderboard of the Information Extraction Task, Robust Reading Competition. https://rrc.cvc.uab.es/?ch=13\&com=evaluation\&task=3 (accessed April 7, 2020) (2020)

14. Ishitani, Y.: Model-based information extraction method tolerant of ocr errors for document images. Int. J. Comput. Process. Orient. Lang. 15, 165-186 (2002)

15. Katti, A.R., Reisswig, C., Guder, C., Brarda, S., Bickel, S., Höhne, J., Faddoul, J.B.: Chargrid: Towards understanding 2D documents. In: EMNLP (2018)

16. Lewis, D., Agam, G., Argamon, S., Frieder, O., Grossman, D., Heard, J.: Building a test collection for complex document information processing. In: Proceedings of the 29th Annual International ACM SIGIR Conference on Research and Development in Information Retrieval (2006)

17. Liu, X., Gao, F., Zhang, Q., Zhao, H.: Graph convolution for multimodal information extraction from visually rich documents. In: NAACL-HLT (2019)

18. Liu, Y., Ott, M., Goyal, N., Du, J., Joshi, M., Chen, D., Levy, O., Lewis, M., Zettlemoyer, L., Stoyanov, V.: RoBERTa: A Robustly Optimized BERT Pretraining Approach. ArXiv 1907.11692 (2019)

19. Medvet, E., Bartoli, A., Davanzo, G.: A probabilistic approach to printed document understanding. IJDAR 14, 335-347 (12 2011)

20. Microsoft: Cognitive Services. https://azure.microsoft.com/en-us/services /cognitive-services/ (accessed November 25, 2019) (2019)

21. Park, S., Shin, S., Lee, B., Lee, J., Surh, J., Seo, M., Lee, H.: CORD: A Consolidated Receipt Dataset for Post-OCR Parsing. In: Document Intelligence Workshop at Neural Information Processing Systems (2019)

22. Peanho, C., Stagni, H., Silva, F.: Semantic information extraction from images of complex documents. Applied Intelligence 37, 543-557 (12 2012)

23. Raffel, C., Shazeer, N., Roberts, A., Lee, K., Narang, S., Matena, M., Zhou, Y., Li, W., Liu, P.J.: Exploring the limits of transfer learning with a unified text-to-text transformer. Journal of Machine Learning Research 21(140), 1-67 (2020) 
24. Rahman, W., Hasan, M., Lee, S., Zadeh, A., Mao, C., Morency, L.P., Hoque, E.: Integrating multimodal information in large pretrained transformers. In: ACL (2020)

25. Rusinol, M., Benkhelfallah, T., Poulain d'Andecy, V.: Field extraction from administrative documents by incremental structural templates. In: ICDAR (2013)

26. Shaw, P., Uszkoreit, J., Vaswani, A.: Self-attention with relative position representations. In: NAACL-HLT (2018)

27. Stanisławek, T., Graliński, F., Wróblewska, A., Lipiński, D., Kaliska, A., Rosalska, P., Topolski, B., Biecek, P.: Kleister: A novel task for information extraction involving long documents with complex layout. ArXiv (accepted to ICDAR 2021) 2105.05796 (2021)

28. Vaswani, A., Shazeer, N., Parmar, N., Uszkoreit, J., Jones, L., Gomez, A.N., Kaiser, Ł., Polosukhin, I.: Attention is all you need. In: Advances in Neural Information Processing Systems 30 (2017)

29. Wang, A., Singh, A., Michael, J., Hill, F., Levy, O., Bowman, S.R.: GLUE: A multitask benchmark and analysis platform for natural language understanding. In: Proceedings of ICLR (2019), https://gluebenchmark.com/ (accessed November 26, 2019)

30. Wolf, T., Debut, L., Sanh, V., Chaumond, J., Delangue, C., Moi, A., Cistac, P., Rault, T., Louf, R., Funtowicz, M., Davison, J., Shleifer, S., von Platen, P., Ma, C., Jernite, Y., Plu, J., Xu, C., Scao, T.L., Gugger, S., Drame, M., Lhoest, Q., Rush, A.M.: Transformers: State-of-the-art natural language processing. In: Proceedings of the 2020 Conference on Empirical Methods in Natural Language Processing: System Demonstrations. pp. 38-45. Association for Computational Linguistics, Online (Oct 2020), https://www .aclweb.org/anthology/2020.emnlp-demos.6

31. Xu, Y., Xu, Y., Lv, T., Cui, L., Wei, F., Wang, G., Lu, Y., Florencio, D., Zhang, C., Che, W., Zhang, M., Zhou, L.: LayoutLMv2: Multi-modal pre-training for visuallyrich document understanding. arXiv 2012.14740 (2020)

32. Xu, Y., Li, M., Cui, L., Huang, S., Wei, F., Zhou, M.: LayoutLM: Pre-training of text and layout for document image understanding. In: Proceedings of the 26th ACM SIGKDD International Conference on Knowledge Discovery \& Data Mining. p. 1192-1200 (2020)

33. Yu, W., Lu, N., Qi, X., Gong, P., Xiao, R.: PICK: Processing key information extraction from documents using improved graph learning-convolutional networks. In: 2020 25th International Conference on Pattern Recognition (ICPR). pp. 43634370 (2021). https://doi.org/10.1109/ICPR48806.2021.9412927

34. Zhang, P., Xu, Y., Cheng, Z., Pu, S., Lu, J., Qiao, L., Niu, Y., Wu, F.: TRIE: End-to-end text reading and information extraction for document understanding. Proceedings of the 28th ACM International Conference on Multimedia (2020) 